( $p<0.01)$, urinary electrolyte excretion $(\mathrm{Na}, \mathrm{Cl}: \mathrm{p}<0.01, \mathrm{~K}: \mathrm{p}<0.05)$, and their clearance (Na:p<0.01, K:p<0.05) were increased. Renal vascular resistance was reduced $(p<0.01)$. No adverse reactions were observed.

The augmented renal blood flow and profound natriuresis after TA-8704 were comparable with those of parenteral dopamine. Our results confirmed beneficial cardiorenal effects of an orally active TA-8704 in patients with severe chronic congestive heart failure. However, further studies on the evaluation of chronic administration of the drug are needed.

\title{
$-240-$ \\ HEMODYNAMIC AND ANTIPLATELET EFFECTS OF PGI2 ANALOG (OP-41483) IN PATIENTS WITH CONGESTIVE HEART FAILURE
}

Yoshiki Takatsu, Yoshiki Yui, Ryuichi Hattori, Keiji Sakaguchi, Takashi Suzawa, Natsuko Yui, Mamoru Takahashi, Takeshi Aoyama and Chuichi Kawai.

Third Division, Department of Internal Medicine, Faculty of Medicine, Kyoto University.

Prostaglandin I2(PGI2) is known to have a strong vasodilating effect as well as an antiplatelet effect. In the previous paper, we have demonstrated salutary effects of PGI2 in patients with severe congestive heart failure using it as a vasodilating agent. However, because PGI2 is a very unstable substanse, there are difficulties in its clinical use. In this study, we evaluated hemodynamic and antiplatelet effects of a newly synthesized, stable analog of PGI2, OP-41483(OP). Its effects were compared with nitroprusside(NP) and PGI2 in patinets with severe congestive heart failure using a Swan-Ganz cathter and a standard platelet aggregation test by 3 uM ADP. In a group of nine patients with severe congestive heart failure, $O P(20 \mathrm{ng} / \mathrm{kg} / \mathrm{min})$ was infused for one hour and its hemodynamic and antiplatelet effects were measured. After an hour for wash-out, NP(1.0 ug/ $/ \mathrm{kg} / \mathrm{min})$ was subsequently infused for one hour and the measurements were similarly done. In another group of nine patients with severe congestive heart failure, PGI2(22 $\mathrm{ng} / \mathrm{kg} / \mathrm{min})$ was infused for one hour and hemodynamics and platelet aggregation were similarly measured. OP showed salutary hemodynamic effects similar to NP and PGI2. Although NP did not show any inhibitory effect on platelet aggregation, OP intibited ADP aggregation to a similar extent as PGI2. No adverse effects were seen during infusion of either drug. These findings suggest that oP may become a new efficacious regimen with an antithrombotic effect in the management of congestive heart failure, espescially, associated with ischemic heart disease.

$-241-$

EFFECTS OF NEW INOTROPIC AGENT, OPC8212, ON AORTIC INPUT IMPEDANCE AND VENOUS RETURN PROPERTY IN ACUTE OPEN CHEST DOGS

Yasuhiko Harasawa, Yoshiaki Nose, and Motoomi Nakamura

Kiyoshi Hayashida, Kenji Sunagawa,

Research Institute of Angiocardiology and Cardiovascular Clinic, and Information Science Laboratory for Biomedicine,
Faculty of Medicine, Kyushu University.

While positive inotropic effects of OPC-8212 (OPC) have been extensively studied, whether its effects on the vascular system are advantageous or not in treating patients with congestive or not in treating patlents with congestive evaluated in 6 dogs effects of OPC on the arterial system properties with Windkessel parameters (total vascular resistance (TVR), arterial compliance (Ca) and characteristic impedance (Rc)) which were derived from high resolution $(0.0122 \mathrm{~Hz})$, wide band $(0.0122-20 \mathrm{~Hz})$ aortic input impedance. We evaluated in another 9 dogs its effects on the venous system 9 dogs its effects on the venous system pressure, conductances of venous return and vascular compliances in systemic and pulmonary vascular bed. We administered $3 \mathrm{mg} / \mathrm{kg}$ opC in each experiment. Administration of OPC increased cardiac output by $53 \%$ [1303 386 (meantsem) to $1709 \pm 294 \mathrm{ml} / \mathrm{min}(\mathrm{p}<0.05)]$ and aortic pressure by $10 \%[90.5 \pm 4.8$ to $81.7 \pm 7.1$ mmHg (statistically insignificant)]. In the arterial system, OPC decreased TVR by $30 \%$

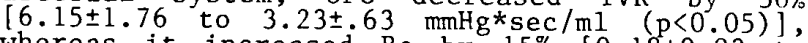
whereas it increased Rc by $15 \%[0.18 \pm 0.03$ to $\left.0.20 \pm 0.03 \mathrm{mmHg}{ }^{*} \mathrm{sec} / \mathrm{ml}(\mathrm{p}<0.05)\right]$ without changing $\mathrm{Ca}[0.42 \pm 0.06$ to $0.43 \pm 0.05 \mathrm{ml} / \mathrm{mmHg}]$. In the varous system, OPC affected none of the

Since OPC decreased afterload without changing preload, we conclude that OPC as an inotropic agent has favorable vascular effects in treating patients with congestive heart failure to increase cardiac output.

$-242-$

\section{ACUTE AND CHRONIC EFFECTS OF XAMOTEROL(CORWIN) IN IDIOPATHIC DILATED CARDIOMYOPATHY}

Kenichi Watanabe, Yoichi Hi rokawa, Akihiro Yokoyama, Hirotaka Oda, Hideaki Ohtsuka, Kazuo Maeda, Tsuneo Nagai, Takashi Tsuda, Senji Hayashi, Yoshimitsu Yazawa, Yutaka Arai and Akira Shibata.

The First Department of Internal Medicine, Niigata University School of Medicine, Niigata 951 JAPAN

Xamoterol(corwin, a B-partial agonist) was given to 10 patients with idiopathic dilated cardiomyopathy(DCM) in NYHA functional class II and III. The acute and chronic effect of xamoterol was assessed, based on the changes in plasma norepinephrine levels(NE), exercise tolerance measured by multistage bicycle ergometer tests, echocardiography, radionuclide ventriculography, and right heart catheterization. The acute effect was deternined after a single intravenous injection on $0.2 \mathrm{mg} / \mathrm{kg}$ of xamoterol. Exercise heart rate decreased from 117 to 97 beats $/ \mathrm{min}(p<0.01)$ but blood pressure and cardiac index were unaffected. When the drug was given orally at $200 \mathrm{mg} /$ day for 3 months, exercise tolerance increased from $4.2 \pm 2.3$ min to $6.4 \pm 2.6$ $\min (\mathrm{p}<0.01)$, echocardiographic ejection fraction(EF) from $29 \pm 12 \%$ to $39 \pm 14 \%(p<0.01)$, and radionuclide EF from $36 \pm 11 \%$ to $45 \pm 14 \%(p<0.01)$, while pulmonary wedge pressure during exercise decreased from $32 \pm 12 \mathrm{mmHg}$ to $22 \pm 9 \mathrm{mmHg}(p<0.05)$. Plasma NE levels which were high $(>0.6 \mathrm{ng} / \mathrm{ml})$ in 3 patients before treatment showed a fall after treatment.

In conclusion, xamoterol exerted both $\beta$-bloking and stimulating effects in a well-balanced fashion, and improved subjective symptoms, exercise tolerance, and parameters of the left ventricle in patients with DCM when used over a long period.

$-243-$

STUDIES OF DENOPAMINE (TA-064) FOR TREATMENT OF REFRACTORY AND/OR BRADYCARDIAC HEART FAILURE

Shigeki Baba, Tomohide Sato, Osamu Tamura, Masahiko Takeshi, Masaaki Kaise, Masanao Shibuya, Yasuro Ishikawa, Keiichi Kusano, Akito Koizumi.

Japanese Circulation Journal Vol. so, June 1986 